\title{
Evaluation of Pre-Released Bold Seeded Lentil Varieties on Different Weather Factors for Growth and Yield Potentiality in the New Alluvial Zone of West Bengal, India
}

\author{
Milan Kanti Kundu*, Sanjib Kumar Mandi, M.D. Hasim Reja, \\ Srijani Maji and Rajib Nath
}

\author{
Department of Agronomy, Bidhan Chandra Krishi Viswavidyalaya, Mohanpur-741252, \\ Nadia, West Bengal, India \\ *Corresponding author
}

\begin{tabular}{|c|c|}
\hline & A B S T R A C T \\
\hline & \multirow{6}{*}{$\begin{array}{l}\text { A study was conducted for two consecutive years }(2012-2013 \text { and } 2013-2014) \text { to assess the } \\
\text { performances of sixteen pre-released and two standard varieties of bold seeded lentil on } \\
\text { different weather factors, for their growth and productivity potential. Growing Degree } \\
\text { Days (GDD) requirement for vegetative stage differ from } 715.8 \text { to } 1077.3 \text { day degree } \\
\text { Celsius, in first year and } 667.7 \text { to } 698.9 \text { day degree Celsius in second year. For completion } \\
\text { of reproductive stage GDD requirement was } 513.5 \text { to } 1016.8 \text { day degree Celsius in first } \\
\text { year and } 644.6 \text { to } 1056.9 \text { day degree Celsius in second year. Heliothermal Unit (HTU) } \\
\text { requirement for vegetative stage differ from } 5343.6 \text { to } 7358.7 \text { day degree Celsius hour in } \\
\text { first year and } 4737.8 \text { to } 4777.8 \text { day degree Celsius hour in second year. HTU requirement } \\
\text { also differ in two years. In first year HTU requirement was } 4076.5 \text { to } 7300.0 \text { day degree } \\
\text { Celsius hour and } 5987.9 \text { to } 11803.7 \text { day degree Celsius hour in second year. Significant } \\
\text { differences were observed in plant height, dry matter accumulation, yield and yield } \\
\text { attributes of the crop. The dry matter accumulation was better in the second year. The seed } \\
\text { yield was maximum in PL- } 129 \text { in both the year. The second year yield was lower than the } \\
\text { first year, as it was exposed to higher temperature during the reproductive phase of the } \\
\text { crop. The maximum productivity of lentil would be archived when the maximum and } \\
\text { minimum temperature during } 100 \% \text { flowering ranged from } 24.6 \text { to } 28.6 \text { and } 10.1 \text { to } 10.9^{\circ} \mathrm{C} \\
\text { in two years respectively. }\end{array}$} \\
\hline & \\
\hline $\begin{array}{l}\text { Pre-released } \\
\text { variety, Lentil, } \\
\text { GDD, HTU, } \\
\text { Yield attributes. }\end{array}$ & \\
\hline Article Info & \\
\hline $\begin{array}{l}\text { Accepted: } \\
\text { 14 July } 2017 \\
\text { Available Online: } \\
\text { 10 September } 2017\end{array}$ & \\
\hline & \\
\hline
\end{tabular}

\section{Introduction}

Lentil is a cool season food legume growing in the span of November to March in West Bengal and rank next only after chick pea in India. The New Alluvial zone of West Bengal is marked by short winter and mild temperature. Because of the temperature sensitivity of this crop, selection of proper varieties of lentil is important for better productivity of this crop. Bhattacharyya
(2009) assessed several lentil genotypes for their yield variability. It is observed that the duration from sowing to flowering is an important parameter for yield variability in pulses. He and Rajaram (1993) observed that the plant height is more sensitive to the temperature in winter crop. However the impact of temperature and Bright sunshine hour (BSSH) on lentil varieties has not been 
evaluated elaborately in the New Alluvial zone of West Bengal. The present experiment has been framed to address this vacuum. It was reported that the local environment important for growth and productivity (Erskin et al., 1989). The major constrains to seed production in most of the cases is higher temperature during flowering and seed growth. Summerfield et al., (1985) reported that higher temperature during reproductive phase accelerated progress towards reproductive maturity and reduce seed yield. Hundal and Kaur (2007) observed that an increase in temperature up to $1{ }^{\circ} \mathrm{C}$ reduces the yield of wheat.

\section{Materials and Methods}

The experiment was carried out during winter (November-March) seasons of 2012-13 and 2013-14 at the District Seed Farm, AB block, Kalyani, BCKV, (Latitude 22 $58^{\prime} \mathrm{N}$ and Longitude $88^{\circ} 32^{\prime} \mathrm{E}$ ), West Bengal, India. The study site is flat and is located at an altitude of $9.75 \mathrm{~m}$ above mean sea level (AMSL). The experimental site falls under tropical humid climate, experiences three distinct seasons (summer, rainy and winter). The mean annual temperature falls below $20^{\circ} \mathrm{C}$ in November and continued up to early part of February. The maximum and minimum relative humidity (RH) ranged from $85-100 \%$ and 22 $96 \%$ respectively. The soil contents $51 \%$ sand, $18.7 \%$ silt and $30.4 \%$ clay and is classified as sandy loam. The $\mathrm{pH}$ of the soil is 7.35 having total nitrogen, available phosphorus, available potassium and organic carbon as $298 \mathrm{~kg} \mathrm{ha}{ }^{-1}, 30.2 \mathrm{~kg} / \mathrm{ha}^{-1}, 195 \mathrm{~kg} / \mathrm{ha}^{-1}$ and $0.48 \%$ respectively. The experiment was conducted in the winter season of 2012-2013 and 2013-2014 in a RBD (Randomised block design) with sixteen pre released bold seeded varieties and two check varieties (K-75, DPL62) for their growth and productivity. Each treatment was allotted in a plot of $4 \times 2.0 \mathrm{~m}$ with two replications. Each treatment received 20, 40 and $40 \mathrm{~kg} \mathrm{~N}, \mathrm{P}_{2} \mathrm{O}_{5}$ and $\mathrm{K}_{2} \mathrm{O}$ per ha through urea, SSP (Single super phosphate) and MOP (Muriate of potash). The description of pre released lentil varieties is given in table 1 . The duration of the different phenophases, plant height, dry matter accumulation, yield attributes and yield were estimated. The growing degree days (GDD) par day was calculated in accordance with the following formula:

$\mathrm{GDD}=\left\{\left[\right.\right.$ Maximum temperature $\left(0^{0} \mathrm{C}\right)+$ Minimum temperature $\left.\left.\left(0^{0} \mathrm{C}\right)\right] / 2\right\}$-Base temperature

Heliothermal unit (day degree Celsius hour) was measured by following formula:

$\mathrm{HTU}=$ GDD of a phenophase $\mathrm{x}$ Average Bright Sunshine hours of that respective phenophase

\section{Results and Discussion}

\section{Impact of weather on growth}

\section{Impact of temperature}

GDD, which is a temperature based index has been computed and its impact on plant height and dry matter accumulation has been assessed. Plant height was significantly affected by the GDD. Plant height increased exponentially with the increment of GDD in all the varieties/genotypes (Fig: 1 ). $\mathrm{R}^{2}$ value ranged from 0.676 (in case of genotype RVL 48) to 0.9036 (in case of genotype LL 1210) indicating the impact of GDD on plant height (Fig 1). GDD requirement for different varieties/genotypes differs (Table 2). The total GDD requirement was maximum in VL 521 in both the years. However, year to year variations were evident because of the temperature variation as well as the difference in the duration in two different years. GDD requirement for the vegetative phase in $1^{\text {st }}$ 
year ranged from 715.8 day $^{\circ} \mathrm{C}$ to 1077.3 day ${ }^{\circ} \mathrm{C}$ where as in the $2^{\text {nd }}$ year, it ranged from 667.7 to 698.9 day ${ }^{\circ}$ C. During reproductive phase the GDD requirement for the $1^{\text {st }}$ and $2^{\text {nd }}$ year ranged from 513.5 to $1016^{\circ} \mathrm{C}$ GDD and 644.6 to $1056.9^{\circ} \mathrm{C}$ GDD respectively (Table 3 ). In the $2^{\text {nd }}$ year the GDD requirement was higher than the $1^{\text {st }}$ year, due to the variation in temperature and duration of the crop.

The dry matter accumulation was found to be the exponential function of GDD although the relationship was insignificant in most of the cases (Fig. 2).

\section{Impact of temperature and BSSH (Bright Sunshine Hour)}

In both the year wide variation in the HTU requirement for the different phenophases was observed for different varieties/genotypes (Table 4). In both the years the genotype VL 521 required maximum HTU from sowing to maturity. The HTU requirement ranged from 9840.5 to 13503.6 day degree Celsius hour in the $2^{\text {nd }}$ year while in the $1^{\text {st }}$ year it ranged from 9887.1 to 14714.2 day degree Celsius hour. In both the year wide variation in the HTU requirement for the onset of different phenophases was observed for different varieties/genotypes (Table 5). HTU requirement for the vegetative and reproductive phases in lentil varied remarkably in two different experimental years. This was due to the variation in the day temperature as well as the sun shine hour in different phenophases. More over the difference in the duration also caused this sort of variation. In the $1^{\text {st }}$ year, the HTU requirement in vegetative phase was higher than the reproductive phase except in case of the genotypes VL 521 and VL 48. Whereas in the $2^{\text {nd }}$ year, the HTU requirement for the reproductive phase was higher than the vegetative phase in most of the cases. This contradictory result appeared due to the variation in the duration of reproductive phase as well as the ambient temperature during the reproductive phase.

The HTU requirement significantly affected the plant height in all the varieties/genotypes. The relationship was found to be exponential. In other words, the plant height in lentil is an exponential function of HTU (Fig. 3). The effect of HTU on dry matter accumulation in different varieties/genotypes was found to be insignificant (Fig. 4).

\section{Summarizing the results the following conclusion may be drawn}

The GDD requirements for the onset of different phenophases differed in different varieties/genotypes. Even the same variety/genotype recorded different GDD requirement in a particular phenophase in two different experimental years. This was due to the variation in the duration and temperature condition in two different years.

Alam et al., (2004) also reported that the GDD requirement varied in different years because of the difference in the atmospheric temperature and the duration of the crop.

Nath et al., (1999) also reported the similar result in sesamum.

Parya et al., (2009) observed that the GDD might significantly predict the stem weight of wheat crop at the milking stage.

The HTU requirement recorded a variation in different varieties/genotypes in two different experimental years. HTU requirements for the vegetative and reproductive phases varied in two different years even for the same genotypes or varieties.

Both the GDD and HTU significantly affected the plant height. However, they did not affect the dry matter accumulation. The relationship was exponential. 
Fig.1 Effect of growing degree days (GDD) on the plant height of the lentil genotypes / varieties

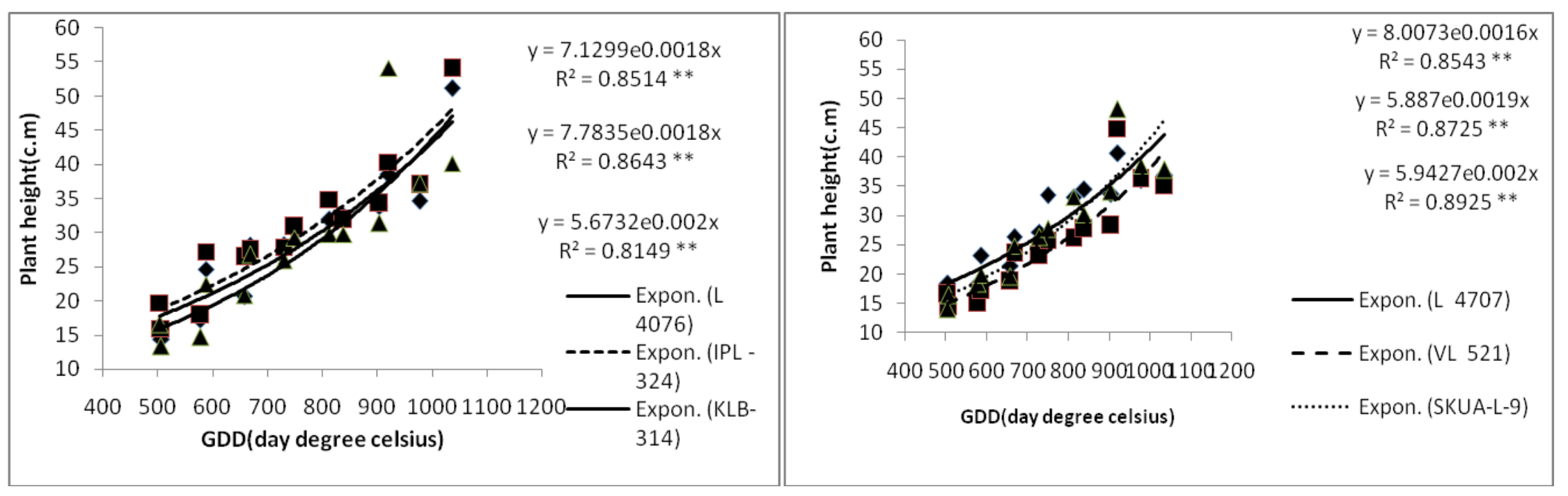

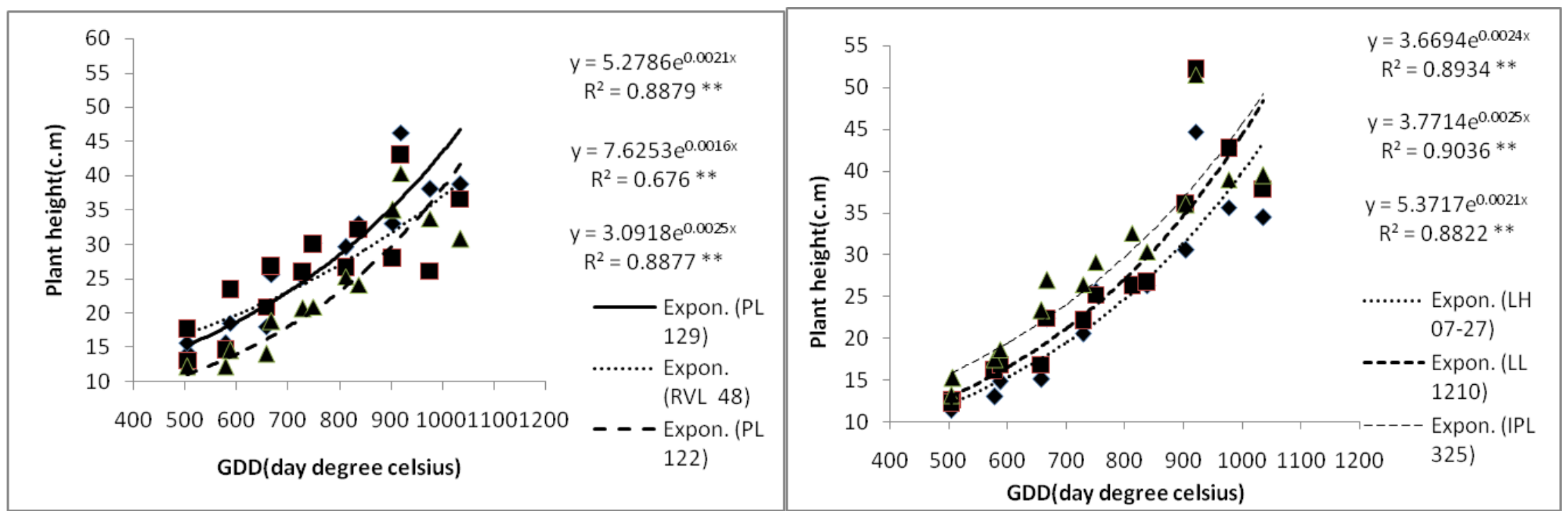



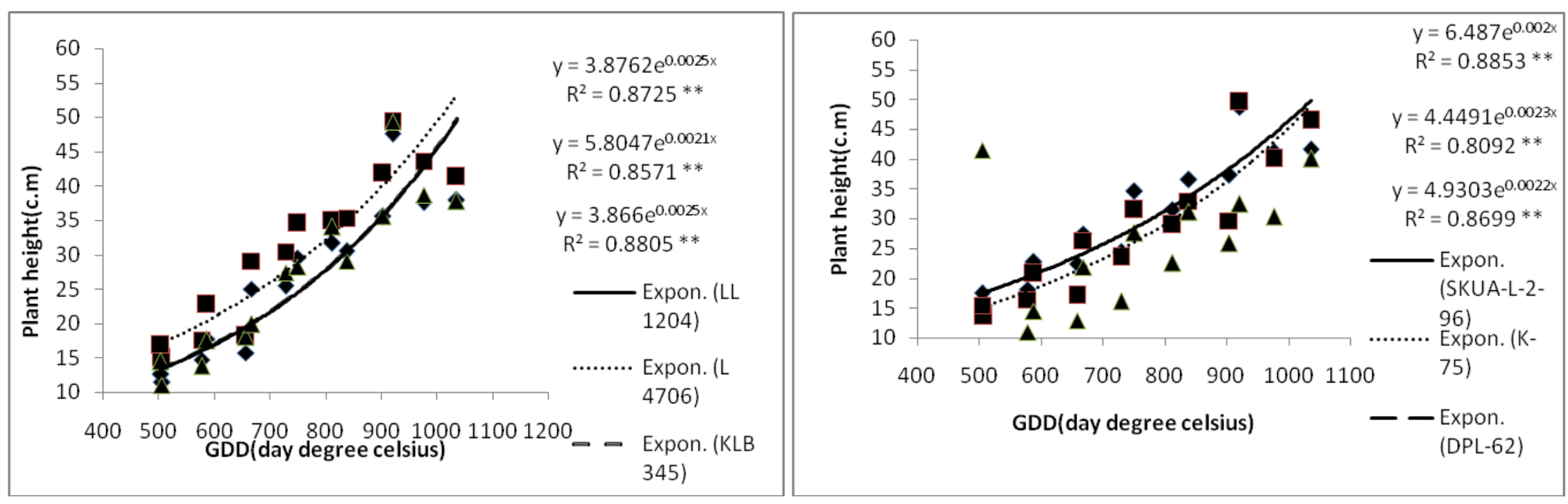

Fig.2 Effect of growing degree days (GDD) on the dry matter accumulation of the lentil genotypes / varieties
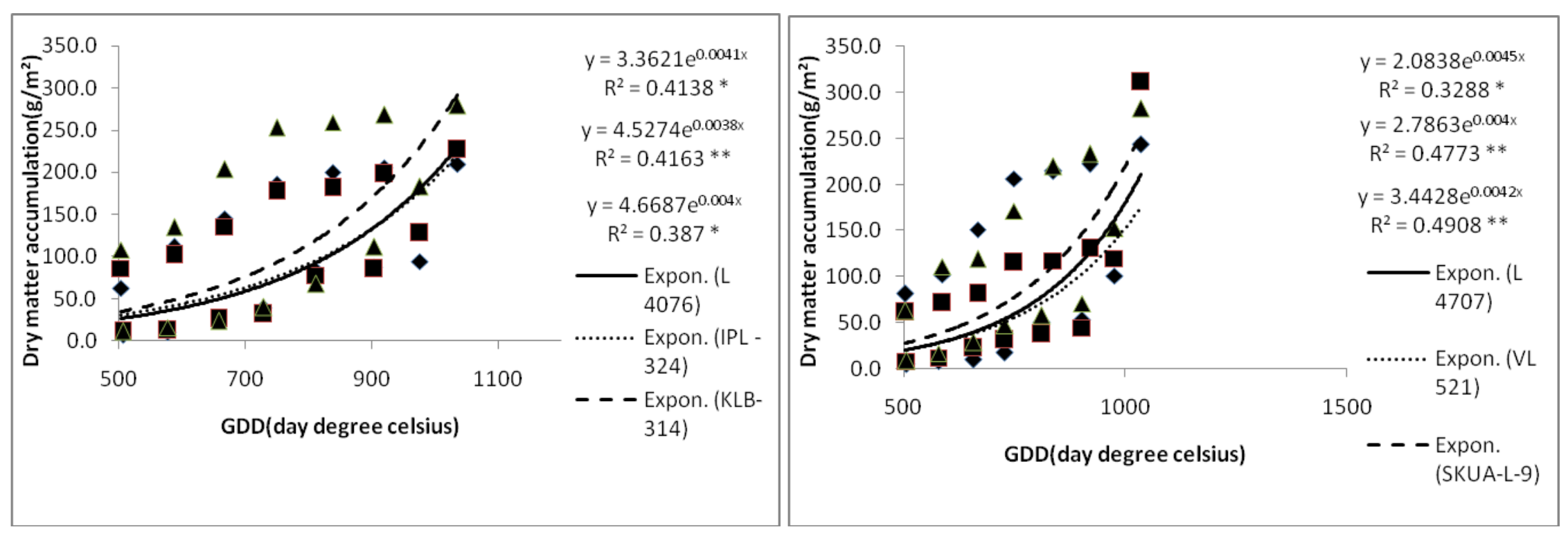
Int.J.Curr.Microbiol.App.Sci (2017) 6(9): 758-771

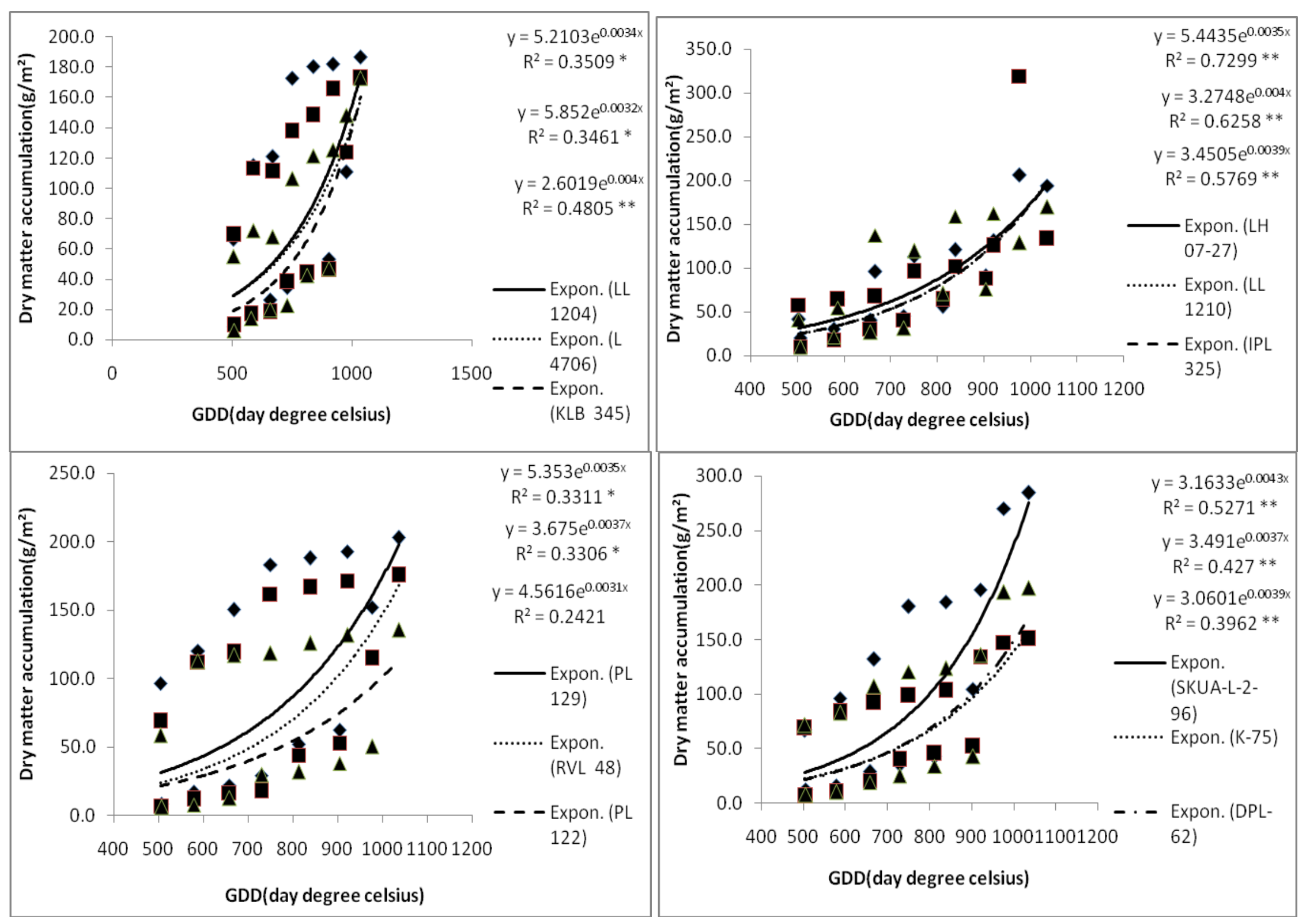


Fig.3 Effect of Heliothermal Unit (HTU) on the plant height of the lentil genotypes / varieties

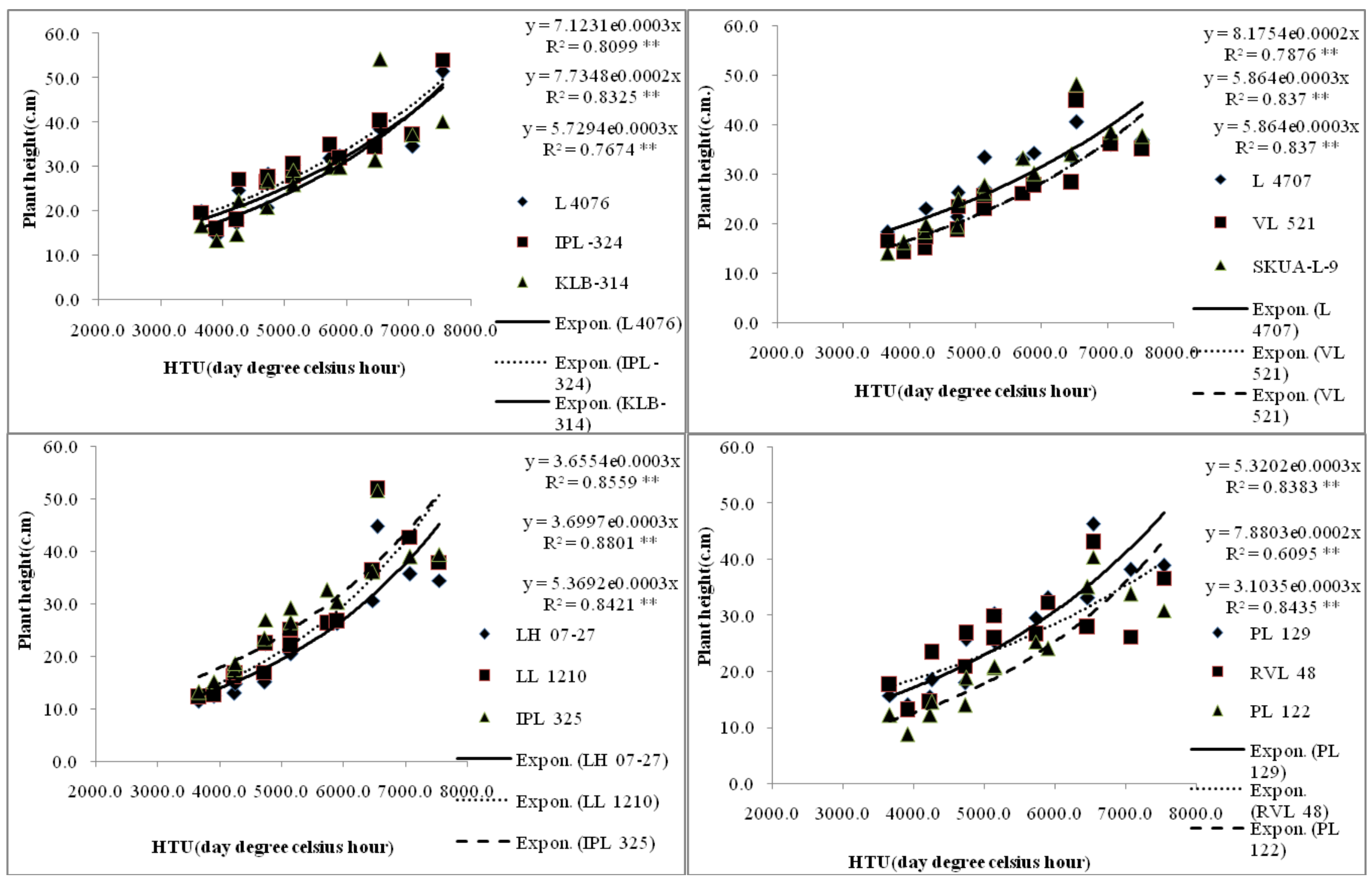




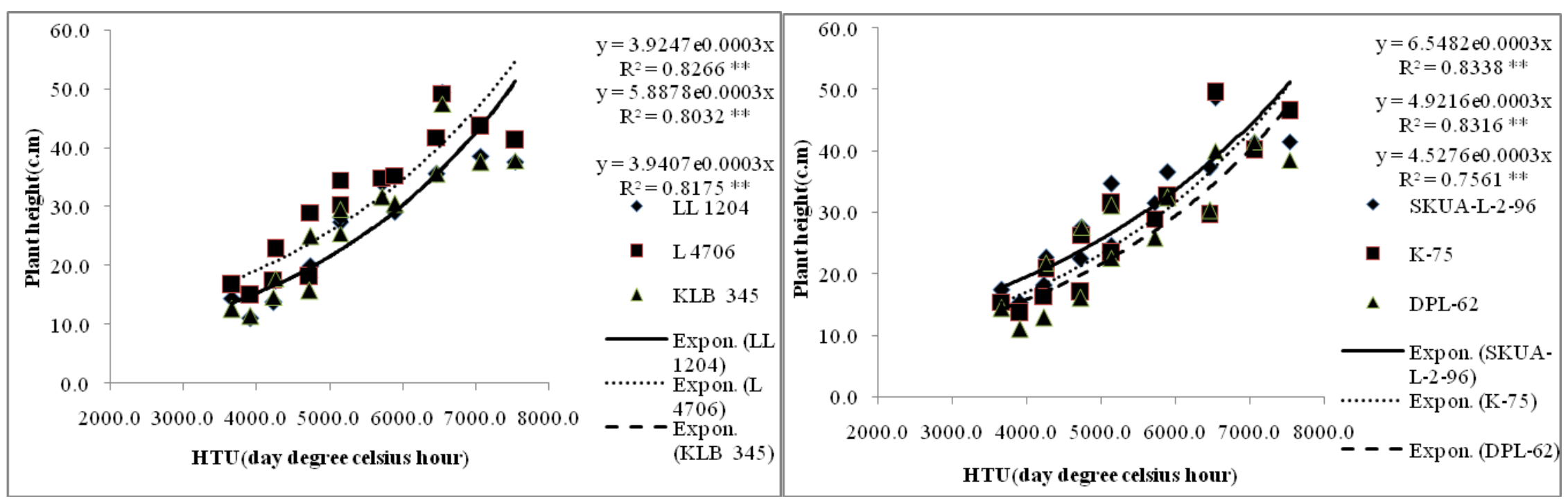

Fig.4 Effect of Heliothermal Unit (HTU) on the dry matter accumulation of the lentil genotypes/varieties

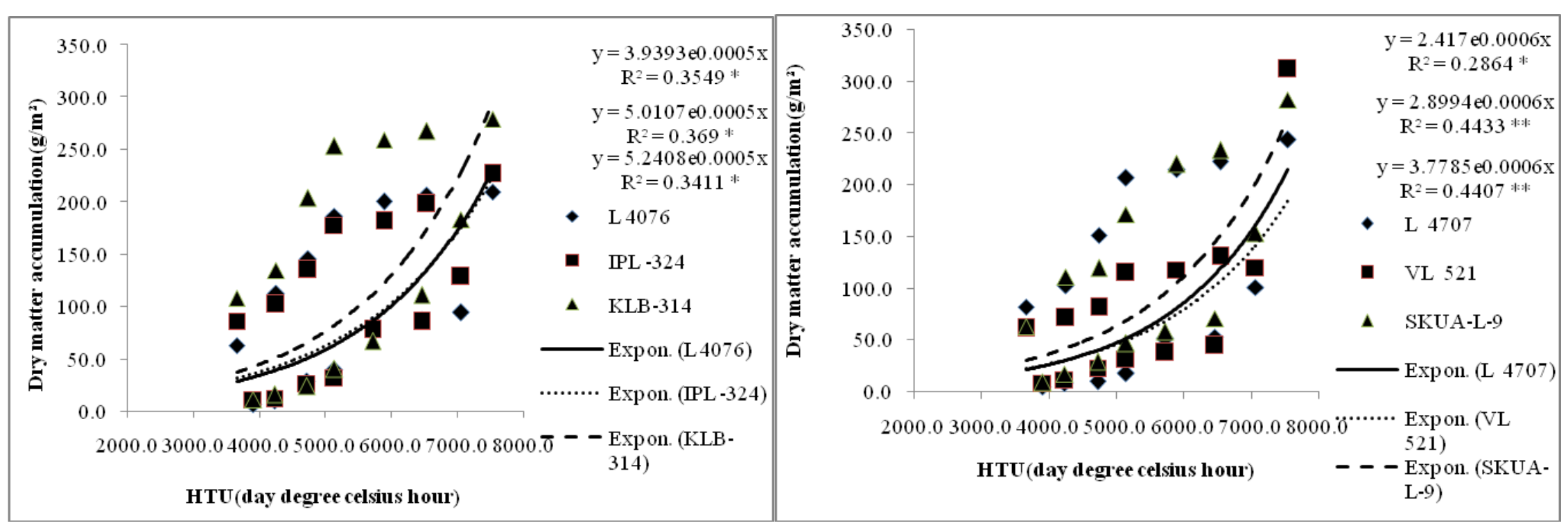




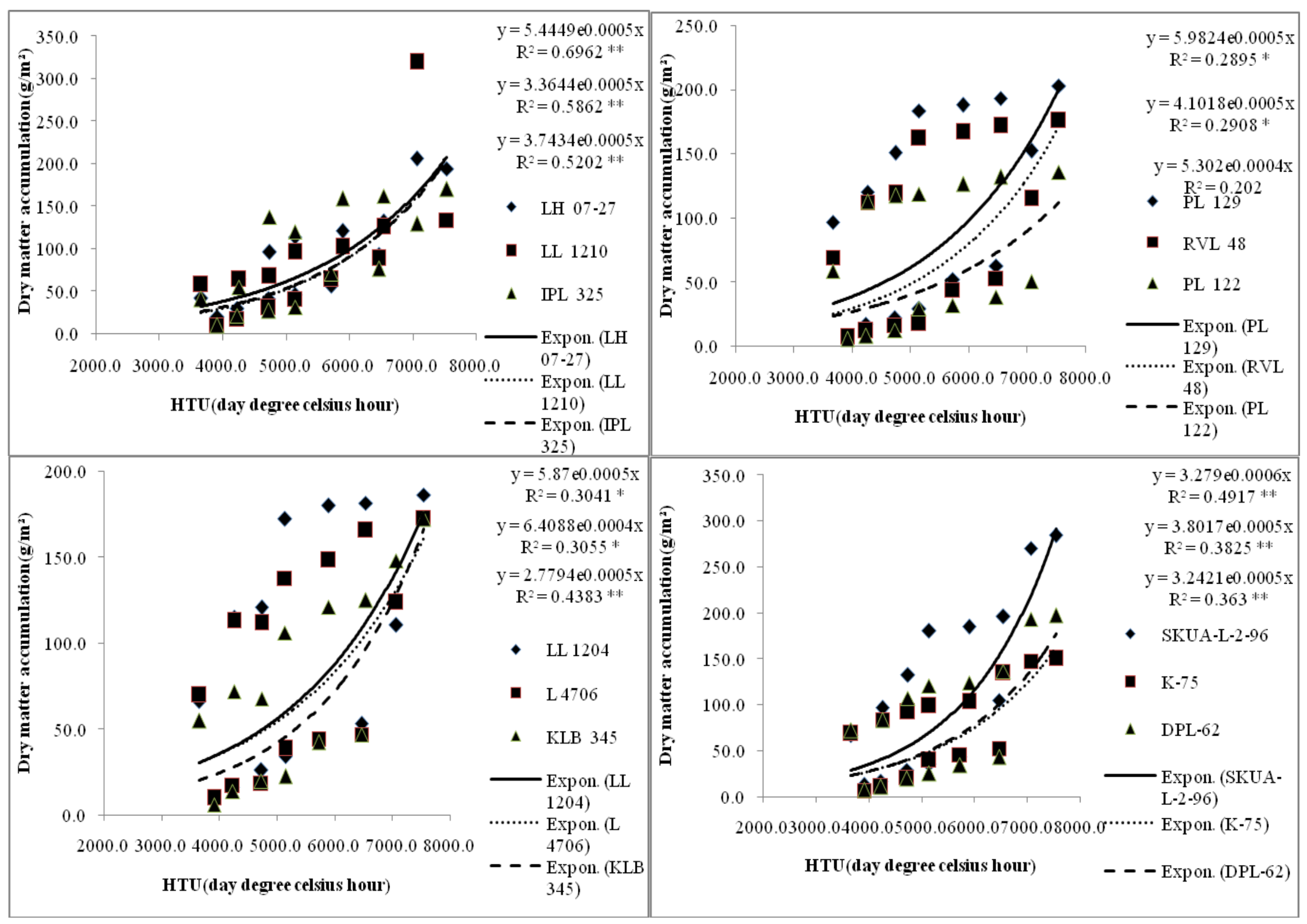


Table.1 Description of lentil genotypes and varieties used in the experiment

\begin{tabular}{|c|c|c|c|}
\hline Sl. No. & Entry & Source & Pedigree \\
\hline 1 & $\mathrm{~L} 4076$ & IARI, New Delhi & Pl 234 x PL 639 \\
\hline 2 & IPL -324 & IIPR, Kanpur & (IIL 7659 X DPL 58) X KL-178 \\
\hline 3 & KLB-314 & CSA, Kanpur & KL 225 X KLB 97-6 \\
\hline 4 & L 4707 & IARI, New Delhi & L 4650 X L 4076 \\
\hline 5 & VL 521 & VPKAS, Almora & VL 501X (Precoz x L 4076) \\
\hline 6 & SKUA-L-9 & Srinagar & Sel. From EC 3109 \\
\hline 7 & LH 07-27 & CCS HAU, Hisar & LH 84-8 X L 4641 \\
\hline 8 & LL 1210 & PAU, Ludhiana & LL 699 X FLIP $91-51$ L \\
\hline 9 & IPL 325 & IIPR, Kanpur & (IIL 101 X EC 362) X DPL 62 \\
\hline 10 & PL 129 & Pantnagar & PL 639 X L 4188 \\
\hline 11 & RVL 48 & Sehore & JL 1 X DPL 62 \\
\hline 12 & PL 122 & Pantnagar & PL 01 X FLIP $96-51$ \\
\hline 13 & LL 1204 & PAU, Ludhiana & LL 148 X DPL 58 \\
\hline 14 & L 4706 & IARI, New Delhi & PL 234 X PL 639 \\
\hline 15 & KLB 345 & CSA, Kanpur & Precoz X KLB 231 \\
\hline 16 & SKUA-L-2-96 & Srinagar & KLF 221 X L 1 \\
\hline 17 & K-75 & CSAU, Kanpur & Sel. From Bundelkhand Local \\
\hline 18 & DPL-62 & IIPR, Kanpur & JL 1 x LG 171 \\
\hline
\end{tabular}

Table.2 GDD Requirement of lentil genotypes/varieties in different phenophases

(Day degree Celsius), 2012-2013 and 2013-2014

\begin{tabular}{|c|c|c|c|c|c|c|c|c|c|c|c|c|}
\hline \multirow{2}{*}{$\begin{array}{c}\text { Genotypes/ } \\
\text { varieties }\end{array}$} & \multicolumn{2}{|c|}{$\begin{array}{c}\text { Sowing- } \\
\text { Germination }\end{array}$} & \multicolumn{2}{|c|}{$\begin{array}{c}\text { Sowing-branch } \\
\text { initiation }\end{array}$} & \multicolumn{2}{|c|}{$\begin{array}{l}\text { Sowing-flower } \\
\text { initiation }\end{array}$} & \multicolumn{2}{|c|}{$\begin{array}{c}\text { Sowing-50\% } \\
\text { flowering }\end{array}$} & \multicolumn{2}{|c|}{$\begin{array}{c}\text { Sowing-100\% } \\
\text { flowering }\end{array}$} & \multicolumn{2}{|c|}{ Sowing-maturity } \\
\hline & 2012-13 & 2013-14 & 2012-13 & \begin{tabular}{|l|}
$2013-14$ \\
\end{tabular} & 2012-13 & 2013-14 & 2012-13 & 2013-14 & 2012-13 & 2013-14 & 2012-13 & 2013-14 \\
\hline L 4076 & 103.1 & 130.4 & 297.3 & 256.4 & 729.1 & 688.8 & 913.5 & 848.6 & 1048.9 & 1096.6 & 1348.1 & \begin{tabular}{|l|}
1596.0 \\
\end{tabular} \\
\hline IPL - 324 & 121.3 & 88.1 & 297.3 & 242.3 & 729.1 & 698.9 & 913.5 & 848.6 & 1026.7 & 1096.6 & 348.1 & 576.2 \\
\hline KLB-314 & 41.6 & 119.0 & 326.3 & 256.4 & 897.3 & 688.8 & 956.3 & 884.3 & 1133.2 & 1140.7 & 410.8 & 91.8 \\
\hline L 4707 & 121.3 & 103.4 & 197.3 & 197.3 & 715.8 & 667.7 & 929.5 & 859.7 & 1037.7 & 1080.5 & 1348.1 & 1438.5 \\
\hline VL 521 & 5.1 & 103.4 & 283.3 & 270.7 & 854.0 & 688.8 & 1088.6 & 884.3 & 1277.5 & 1140.7 & 1870.8 & 745.6 \\
\hline SKUA-L-9 & 103.1 & 119.0 & 297.3 & 270.7 & 715.8 & 678.0 & 956.3 & 872.2 & 1048.9 & 1112.5 & 1410.8 & 338.5 \\
\hline LH 07-27 & 103.1 & 135.6 & 297.3 & 297.9 & 913.5 & 667.7 & 1088.6 & 872.2 & 1294.4 & 1096.6 & 1624.8 & 1657.8 \\
\hline LL 1210 & 141.6 & 119.0 & 326.3 & 84.7 & 805.5 & 688.8 & 1088.6 & 884.3 & 1308.6 & 1126.2 & 1.8 & 1657.8 \\
\hline IPL 32 & 1.3 & 03.4 & 311.9 & 0.7 & 881.7 & 98.9 & 967.6 & 920.7 & 1117.3 & 1165.5 & & 33.2 \\
\hline PL 12 & 5.8 & 03.4 & 297.3 & 34.7 & 814. & 698.9 & 913.5 & 920.7 & 1103.0 & 1165.5 & & 91.8 \\
\hline RVL 4 & 1.3 & 5.6 & 297.3 & 70.7 & 832. & 688.8 & 913.5 & 897.2 & 1037.7 & 1140.7 & & 33.3 \\
\hline PL 12 & 3.1 & 19.0 & 297.3 & 297.9 & 742.9 & 678.3 & 1061.6 & 884.3 & 1260.3 & 1140.7 & & 1596.0 \\
\hline LL 1204 & 3.1 & 03.4 & 297.3 & 284.7 & 832.7 & 667.7 & 1009.4 & 884.3 & 1277.5 & 1152.7 & & 1616.3 \\
\hline L 4706 & 1.3 & 03.4 & 326.3 & 284.7 & 897.3 & 678.3 & 988.9 & 909.0 & 1216.3 & 1126.2 & & 33.3 \\
\hline KLB? & 103.1 & 135.6 & 297.3 & 311.1 & 805.5 & 688.8 & 1007.9 & 872.2 & 1277.5 & 1112.5 & & 1596.0 \\
\hline $\begin{array}{l}\text { SKUA-L-2- } \\
96\end{array}$ & 103.1 & 119.0 & 297.3 & 297.9 & 897.3 & 698.9 & 978.3 & 909.0 & 1277.3 & 1152.7 & 1481.8 & 1657.8 \\
\hline K-75 & 3.1 & 9.0 & 297.3 & 4.7 & 1077.3 & & 1243.7 & 572.2 & 1308.6 & 1152.7 & 1624.8 & 1657.8 \\
\hline DPL-62 & 103.1 & 119.0 & 297.3 & 270.7 & 832.7 & 698.9 & 1088.6 & 884.3 & 1277.5 & 1140.7 & 1624.8 & 1657.8 \\
\hline
\end{tabular}


Table.3 GDD Requirement for the onset of different phenophases in lentil varieties (Day degree Celsius), 2012-2013

\begin{tabular}{|c|c|c|c|c|c|c|c|c|}
\hline $\begin{array}{c}\text { Genotypes/ } \\
\text { varieties }\end{array}$ & $\begin{array}{c}\text { Sowing to } \\
\text { Germination }\end{array}$ & $\begin{array}{c}\text { Germination to } \\
\text { Branch } \\
\text { initiation }\end{array}$ & $\begin{array}{c}\text { Branch } \\
\text { initiation to } \\
\text { flower } \\
\text { initiation }\end{array}$ & $\begin{array}{c}\text { GDD } \\
\text { (Vegetative } \\
\text { phase) }\end{array}$ & $\begin{array}{c}\text { Flower } \\
\text { initiation to } \\
50 \% \\
\text { flowering }\end{array}$ & $\begin{array}{c}50 \% \\
\text { flowering to } \\
100 \% \\
\text { flowering }\end{array}$ & $\begin{array}{c}100 \% \\
\text { flowering to } \\
\text { Maturity }\end{array}$ & $\begin{array}{c}\text { GDD } \\
\text { (Reproducti } \\
\text { ve stage) }\end{array}$ \\
\hline L 4076 & 103.1 & 194.2 & 431.8 & 729.1 & 184.4 & 135.5 & 299.2 & 619.1 \\
\hline IPL -324 & 121.3 & 176.0 & 431.8 & 729.1 & 184.4 & 113.3 & 321.4 & 619.1 \\
\hline KLB-314 & 141.6 & 184.8 & 571.0 & 897.3 & 59.0 & 176.9 & 277.6 & 513.5 \\
\hline L 4707 & 121.3 & 76.0 & 518.6 & 715.8 & 213.7 & 108.2 & 310.4 & 632.3 \\
\hline VL 521 & 85.1 & 198.2 & 570.7 & 854.0 & 234.6 & 188.9 & 593.3 & 1016.8 \\
\hline SKUA-L-9 & 103.1 & 194.2 & 418.6 & 715.8 & 240.5 & 92.6 & 361.9 & 695.0 \\
\hline LH 07-27 & 103.1 & 194.2 & 616.2 & 913.5 & 175.2 & 205.8 & 330.5 & 711.4 \\
\hline LL 1210 & 141.6 & 184.8 & 479.2 & 805.5 & 283.2 & 220.0 & 173.2 & 676.4 \\
\hline IPL 325 & 121.3 & 190.6 & 569.9 & 881.7 & 85.9 & 149.7 & 293.5 & 529.1 \\
\hline PL 129 & 85.8 & 211.5 & 516.8 & 814.1 & 99.4 & 189.6 & 307.8 & 596.8 \\
\hline RVL 48 & 121.3 & 176.0 & 535.5 & 832.7 & 80.8 & 124.3 & 373.1 & 578.1 \\
\hline PL 122 & 103.1 & 194.2 & 445.7 & 742.9 & 318.7 & 198.7 & 364.5 & 881.9 \\
\hline LL 1204 & 103.1 & 194.2 & 535.5 & 832.7 & 176.7 & 268.1 & 133.3 & 578.1 \\
\hline L 4706 & 121.3 & 205.1 & 571.0 & 897.3 & 91.6 & 227.4 & 194.5 & 513.5 \\
\hline KLB 345 & 103.1 & 194.2 & 508.2 & 805.5 & 202.5 & 269.6 & 204.3 & 676.4 \\
\hline SKUA-L-2-96 & 103.1 & 194.2 & 600.1 & 897.3 & 81.0 & 299.0 & 204.6 & 584.5 \\
\hline K-75 & 103.1 & 194.2 & 780.0 & 1077.3 & 166.5 & 64.9 & 316.2 & 547.6 \\
\hline DPL-62 & 103.1 & 194.2 & 535.5 & 832.7 & 255.9 & 188.9 & 347.3 & 792.1 \\
\hline
\end{tabular}

B. GDD Requirement for the onset of different phenophases in lentil varieties (Day degree Celsius), 2013-2014

\begin{tabular}{|c|c|c|c|c|c|c|c|c|}
\hline $\begin{array}{c}\text { Genotypes/ } \\
\text { varieties }\end{array}$ & $\begin{array}{c}\text { Sowing to } \\
\text { Germination }\end{array}$ & $\begin{array}{c}\text { Germination to } \\
\text { Branch } \\
\text { initiation }\end{array}$ & $\begin{array}{c}\text { Branch } \\
\text { initiation to } \\
\text { flower } \\
\text { initiation }\end{array}$ & $\begin{array}{c}\text { GDD } \\
\text { (Vegetative } \\
\text { stage) }\end{array}$ & \begin{tabular}{|c|} 
Flower \\
initiation to \\
$50 \%$ \\
flowering \\
\end{tabular} & $\begin{array}{c}50 \% \\
\text { flowering to } \\
100 \% \\
\text { flowering }\end{array}$ & $\begin{array}{c}100 \% \\
\text { flowering to } \\
\text { Maturity }\end{array}$ & $\begin{array}{c}\text { GDD } \\
\text { (Reproduc } \\
\text { tive stage) }\end{array}$ \\
\hline L 4076 & 130.4 & 126.0 & 432.4 & 688.8 & \begin{tabular}{|l|}
159.8 \\
\end{tabular} & 248.0 & 499.4 & 907.2 \\
\hline IPL -324 & 88.1 & 154.2 & 456.7 & 698.9 & 149.7 & 248.0 & 479.6 & 877.3 \\
\hline KLB-314 & 119.0 & 137.4 & 432.4 & 688.8 & 195.6 & 256.4 & 351.1 & 803.0 \\
\hline L 4707 & 103.4 & 93.9 & 470.5 & 667.7 & 192.0 & 220.9 & 358.0 & 770.8 \\
\hline VL 521 & 103.4 & 167.3 & 418.1 & 688.8 & 195.6 & 256.4 & 604.9 & 1056.9 \\
\hline SKUA-L-9 & 119.0 & 151.7 & 407.6 & 678.3 & 194.1 & 240.4 & 326.0 & 760.4 \\
\hline LH 07-27 & 135.6 & 162.3 & 369.9 & 667.7 & 204.5 & 224.4 & 561.3 & 990.1 \\
\hline LL 1210 & 119.0 & 165.7 & 404.1 & 688.8 & 195.6 & 241.9 & 531.6 & 969.1 \\
\hline IPL 325 & 103.4 & 167.3 & 428.2 & 698.9 & 221.8 & 244.8 & 367.7 & 834.3 \\
\hline PL 129 & 103.4 & 181.3 & 414.2 & 698.9 & 221.8 & 244.8 & 326.3 & 792.9 \\
\hline RVL 48 & 135.6 & 135.2 & 418.1 & 688.8 & 208.4 & 243.6 & 192.6 & 644.6 \\
\hline PL 122 & 119.0 & 178.9 & 380.5 & 678.3 & 206.0 & 256.4 & 455.3 & 917.7 \\
\hline LL 1204 & 103.4 & 181.3 & 383.0 & 667.7 & 216.6 & 268.4 & 463.6 & 948.6 \\
\hline L 4706 & 103.4 & 181.3 & 393.6 & 678.3 & 230.7 & 217.3 & 207.1 & 655.0 \\
\hline KLB 345 & 135.6 & 175.6 & 377.7 & 688.8 & 183.4 & 240.4 & 483.5 & 907.2 \\
\hline SKUA-L-2-96 & 119.0 & 178.9 & 401.1 & 698.9 & 210.1 & 243.8 & 505.1 & 958.9 \\
\hline K-75 & 119.0 & 165.7 & 404.1 & 688.8 & 183.4 & 280.6 & 505.1 & 969.1 \\
\hline DPL-62 & 119.0 & 151.7 & 428.2 & 698.9 & 185.4 & 256.4 & 517.1 & 958.9 \\
\hline
\end{tabular}


Table.4 HTU Requirement of lentil genotypes/varieties in different phenophases (Day degree Celsius hour), 2012-2013 and 2013-2014

\begin{tabular}{|c|c|c|c|c|c|c|c|c|c|c|c|c|}
\hline \multirow{2}{*}{$\begin{array}{c}\text { Genotypes/ } \\
\text { varieties }\end{array}$} & \multicolumn{2}{|c|}{$\begin{array}{c}\text { Sowing- } \\
\text { Germination }\end{array}$} & \multicolumn{2}{|c|}{$\begin{array}{l}\text { Sowing-branch } \\
\text { initiation }\end{array}$} & \multicolumn{2}{|c|}{$\begin{array}{c}\text { Sowing-flower } \\
\text { initiation }\end{array}$} & \multicolumn{2}{|c|}{$\begin{array}{c}\text { Sowing-50\% } \\
\text { flowering }\end{array}$} & \multicolumn{2}{|c|}{$\begin{array}{c}\text { Sowing-100\% } \\
\text { flowering }\end{array}$} & \multicolumn{2}{|c|}{ Sowing-maturity } \\
\hline & 2012-13 & 2013-14 & 2012-13 & 2013-14 & 2012-13 & 2013-14 & $2012-13$ & 2013-14 & $2012-13$ & 2013-14 & 2012-13 & 2013-14 \\
\hline L 4076 & 843.5 & 796.2 & & & & 4771.7 & 6567.8 & 5951.0 & & & 9887.1 & 12114.4 \\
\hline IPL -324 & 977.9 & 658.1 & 2458.0 & & 5436.0 & & 6567.8 & 5951.0 & 7475.6 & 8067.5 & 9887.1 & 11910.4 \\
\hline KLB-314 & 1059.1 & 925.7 & 2724.8 & & 6446.7 & & 6864.2 & 6235.8 & 8267.3 & & 10523.2 & 11243.5 \\
\hline L 4707 & 977.9 & 796.2 & 2457.5 & 1532.3 & 5343.6 & 4737.8 & 6691.0 & 6041.0 & 7580.1 & 7937.5 & 9887.1 & 10758.9 \\
\hline VL 521 & 724.7 & 796.2 & 2332.0 & 2037.1 & 1646.2 & 4771.7 & 7888.4 & 3235.8 & 9472.1 & 8222.8 & & 13503.6 \\
\hline SKUA-L-9 & 843.5 & 925.7 & 2332.0 & 2037.1 & 5343.6 & 4770.7 & 6864.2 & 6142.2 & 7684.3 & 8101.0 & 10523.2 & 10758.6 \\
\hline LH 07-27 & 843.5 & 1081.3 & 2458.0 & 2248.8 & 6568.0 & 4737.8 & 7888.0 & 6142.2 & 9632.7 & 8067.5 & 12747.4 & 12697.3 \\
\hline LL 1210 & 1059.6 & 25.7 & 2724.8 & 2146.3 & 5803.0 & 4771.7 & 7888.4 & 6235.8 & 9639.8 & 8102.4 & 11262.2 & 12697.3 \\
\hline IPL 325 & 977.9 & 796.2 & 2591.9 & 2037.1 & 6328.1 & 4777.8 & 6973.8 & 6538.3 & 8119.4 & 8468.9 & 10523.2 & 11620.0 \\
\hline PL 129 & 843.5 & 796.2 & 2457.6 & 2146.3 & 5868.4 & 4777.8 & 6567.8 & 6538.3 & 8003.2 & 8468.9 & 10523.2 & 11243.5 \\
\hline RVL 48 & 977.9 & 1081.3 & 2457.6 & 2037.0 & 6004.5 & 4777.8 & 6567.8 & 6338.6 & 7580.1 & 8222.7 & 10523.2 & 9840.5 \\
\hline PL 122 & 843.5 & 925.7 & 2457.5 & 2248.8 & 5447.4 & 4770.7 & 7788.4 & 6235.8 & 9298.9 & 8222.8 & 12747.5 & 12114.4 \\
\hline LL 1204 & 843.5 & 796.2 & 2457.5 & 2146.3 & 6004.5 & 4737.8 & 7475.6 & 6235.8 & 9472.1 & 8343.9 & 10523.2 & 12327.5 \\
\hline L 4706 & 977.9 & 796.2 & 2724.8 & 2146.3 & 6446.7 & 4770.7 & 7186.8 & 6436.5 & 8918.3 & 8102.4 & 10523.2 & 9840.5 \\
\hline KLB 345 & 843.5 & 1081.3 & 2457.5 & 2368.1 & 5803.0 & 4771.7 & 7358.7 & 6142.2 & 9472.1 & 8109.0 & 11262.2 & 12114.4 \\
\hline $\begin{array}{c}\text { SKUA-L-2- } \\
96\end{array}$ & 843.5 & 925.7 & 2457.5 & 2248.8 & 6446.7 & 4777.8 & 7090.9 & 6436.5 & 9472.1 & 8343.9 & 11262.2 & 12697.3 \\
\hline K-75 & 843.5 & 925.7 & 2457.5 & 2146.3 & 7358.7 & 4771.7 & 8003.2 & 6142.2 & 9639.8 & 8343.9 & 12747.5 & 12697.3 \\
\hline DPL-62 & 843.5 & 925.7 & 2457.5 & 2146.3 & 6004.5 & 4777.8 & 7888.4 & 6235.8 & 9472.1 & 8222.7 & 12747.5 & 12697.3 \\
\hline
\end{tabular}

Table.5 HTU requirement onset of different phenophases in lentil varieties (Day degree Celsius hour) in $1^{\text {st }}$ year

\begin{tabular}{|c|c|c|c|c|c|c|c|c|}
\hline $\begin{array}{c}\text { Genotypes/ } \\
\text { varieties }\end{array}$ & $\begin{array}{c}\text { Sowing to } \\
\text { Germination }\end{array}$ & $\begin{array}{c}\text { Germination to } \\
\text { Branch } \\
\text { initiation }\end{array}$ & $\begin{array}{c}\text { Branch } \\
\text { initiation to } \\
\text { flower } \\
\text { initiation } \\
\end{array}$ & $\begin{array}{c}\text { HTU } \\
\text { (Vegetative } \\
\text { phase) }\end{array}$ & \begin{tabular}{|c|} 
Flower \\
initiation to \\
$50 \%$ \\
flowering \\
\end{tabular} & \begin{tabular}{|c|}
$50 \%$ \\
flowering to \\
$100 \%$ \\
flowering \\
\end{tabular} & $\begin{array}{c}100 \% \\
\text { flowering to } \\
\text { Maturity }\end{array}$ & $\begin{array}{c}\text { HTU } \\
\text { (Reproductiv } \\
\text { e phase) }\end{array}$ \\
\hline L 4076 & 843.5 & 1614.0 & 2978.8 & 5436.3 & \begin{tabular}{|l|}
1131.5 \\
\end{tabular} & 1116.5 & 2202.8 & 4450.8 \\
\hline IPL -324 & 977.9 & 1480.1 & 2978.0 & 5436.0 & 1131.8 & 907.9 & 2411.5 & 4451.1 \\
\hline KLB-314 & 1059.1 & 1665.8 & 3721.9 & 6446.7 & 417.5 & 1403.1 & 2255.9 & 4076.5 \\
\hline L 4707 & 977.9 & 1479.7 & 2886.0 & 5343.6 & 1347.5 & 889.1 & 2307.0 & 4543.5 \\
\hline VL 521 & 724.7 & 1607.3 & 2965.5 & 5297.5 & 2415.3 & 1583.8 & 2854.6 & 6853.7 \\
\hline SKUA-L-9 & 843.5 & 1488.4 & 3011.6 & 5343.6 & 1520.6 & 820.1 & 2838.9 & 5179.6 \\
\hline LH 07-27 & 843.5 & 1614.5 & 4110.0 & 6568.0 & 1320.0 & 1744.7 & 3114.7 & 6179.4 \\
\hline LL 1210 & 1059.6 & 1665.2 & 3078.2 & 5803.0 & 2085.4 & 1751.4 & 1622.4 & 5459.2 \\
\hline IPL 325 & 977.9 & 1614.0 & 3736.2 & 6328.1 & 645.7 & 1145.6 & 2403.8 & 4195.1 \\
\hline PL 129 & 843.5 & 1614.0 & 3410.8 & 5868.4 & 699.4 & 1435.4 & 2520.0 & 4654.8 \\
\hline RVL 48 & 977.9 & 1479.7 & 3546.9 & 6004.5 & 563.3 & 1012.4 & 2943.1 & 4518.7 \\
\hline PL 122 & 843.5 & 1614.0 & 2989.9 & 5447.4 & 2341.0 & 1510.5 & 3448.5 & 7300.0 \\
\hline LL 1204 & 843.5 & 1614.0 & 3546.9 & 6004.5 & 1471.1 & 1996.5 & 1051.0 & 4518.7 \\
\hline L 4706 & 977.9 & 1747.0 & 3721.9 & 6446.7 & 740.1 & 1731.5 & 1604.9 & 4076.5 \\
\hline KLB 345 & 843.5 & 1614.0 & 3345.5 & 5803.0 & 1555.7 & 2113.4 & 1790.1 & 5459.2 \\
\hline SKUA-L-2-96 & 843.5 & 1614.0 & 3989.1 & 6446.7 & 644.3 & 2381.2 & 1790.1 & 4815.6 \\
\hline $\mathrm{K}-75$ & 843.5 & 1614.0 & 4901.2 & 7358.7 & 644.4 & 1636.6 & 3107.7 & 5388.7 \\
\hline DPL-62 & 843.5 & 1614.0 & 3547.0 & 6004.5 & 1883.9 & 1583.8 & 3275.3 & 6743.0 \\
\hline
\end{tabular}


B. HTU requirement onset of different phenophases in lentil genotypes/varieties (Day degree Celsius hour) in $2^{\text {nd }}$ year

\begin{tabular}{|l|c|c|c|c|c|c|c|c|}
\hline $\begin{array}{c}\text { Genotypes/ } \\
\text { varieties }\end{array}$ & $\begin{array}{c}\text { Sowing to } \\
\text { Germination }\end{array}$ & $\begin{array}{c}\text { Germination to } \\
\text { Branch } \\
\text { initiation }\end{array}$ & $\begin{array}{c}\text { Branch } \\
\text { initiation to } \\
\text { flower } \\
\text { initiation }\end{array}$ & $\begin{array}{c}\text { HTU } \\
\text { (Vegetative } \\
\text { phase) }\end{array}$ & $\begin{array}{c}\text { Flower } \\
\text { initiation to } \\
\mathbf{5 0 \%} \\
\text { flowering }\end{array}$ & $\begin{array}{c}\mathbf{5 0 \%} \\
\text { flowering to } \\
\text { 100\% } \\
\text { flowering }\end{array}$ & $\begin{array}{c}\text { 100\% } \\
\text { flowering to } \\
\text { Maturity }\end{array}$ & $\begin{array}{c}\text { HTU(Repro } \\
\text { ductive } \\
\text { phase) }\end{array}$ \\
\hline L 4076 & 796.2 & 1117.4 & 2858.0 & 4771.7 & 1179.3 & 2116.5 & 4046.9 & 7342.7 \\
\hline IPL -324 & 658.1 & 1142.8 & 2976.9 & 4777.8 & 1173.3 & 2116.5 & 3842.9 & 7132.7 \\
\hline KLB-314 & 925.7 & 988.0 & 2858.1 & 4771.7 & 1464.1 & 1987.0 & 3020.8 & 6471.8 \\
\hline L 4707 & 796.2 & 736.1 & 3205.5 & 4737.8 & 1303.2 & 1896.6 & 2821.3 & 6021.1 \\
\hline VL 521 & 796.2 & 1240.8 & 2734.6 & 4771.7 & 1535.9 & 4987.0 & 5280.8 & 11803.7 \\
\hline SKUA-L-9 & 925.7 & 1111.4 & 2733.6 & 4770.7 & 1371.5 & 1958.8 & 2657.6 & 5987.9 \\
\hline LH 07-27 & 1081.3 & 1167.6 & 2489.0 & 4737.8 & 1404.4 & 1925.3 & 4629.8 & 7959.5 \\
\hline LL 1210 & 925.7 & 1220.6 & 2625.4 & 4771.7 & 1464.1 & 1866.6 & 4594.9 & 7925.6 \\
\hline IPL 325 & 796.2 & 1240.8 & 2740.7 & 4777.8 & 1760.5 & 1930.6 & 3151.1 & 6842.2 \\
\hline PL 129 & 796.2 & 1350.0 & 2631.5 & 4777.8 & 1760.5 & 1930.6 & 2774.6 & 6465.7 \\
\hline RVL 48 & 1081.3 & 955.7 & 2740.8 & 4777.8 & 1560.8 & 1884.1 & 1617.8 & 5062.7 \\
\hline PL 122 & 925.7 & 1323.1 & 2521.8 & 4770.7 & 1465.1 & 1987.0 & 3891.6 & 7343.7 \\
\hline LL 1204 & 796.2 & 1350.0 & 2591.5 & 4737.8 & 1498.0 & 2108.2 & 3983.6 & 7589.7 \\
\hline L 4706 & 796.2 & 1350.0 & 2624.4 & 4770.7 & 1665.9 & 1665.9 & 1738.1 & 5069.8 \\
\hline KLB 345 & 1081.3 & 1286.8 & 2403.6 & 4771.7 & 1370.5 & 1966.8 & 4005.4 & 7342.7 \\
\hline SKUA-L-2-96 & 925.7 & 1323.1 & 2529.0 & 4777.8 & 1658.7 & 1907.4 & 4353.4 & 7919.5 \\
\hline K-75 & 925.7 & 1220.6 & 2625.4 & 4771.7 & 1370.5 & 2201.7 & 4353.4 & 7925.6 \\
\hline DPL-62 & 925.7 & 1220.6 & 2631.5 & 4777.8 & 1458.0 & 1986.9 & 4474.6 & 7919.5 \\
\hline
\end{tabular}

\section{Yield and yield attributes}

Significant differences did exist among the variety regarding the number of branch plant ${ }^{-1}$ and number of pods plant ${ }^{-1}$. The maximum seed yield in both the year was recorded in PL-129. In the $1^{\text {st }}$ year it was $1712.4 \mathrm{~kg} \mathrm{ha}^{-1}$ and in the $2^{\text {nd }}$ year $1602.4 \mathrm{~kg} \mathrm{ha}^{-1}$. The minimum yield was recorded in VL 521 $\left(336.7 \mathrm{~kg} \mathrm{ha}^{-1}\right)$. The highly productive four pre released varieties were PL 129, L 4706, SKUA-L-9 and IPL-325.

The remarkable differences observed in different vegetative growth stage in the two experimental year was due to temperature difference in two different experimental years. In the $2^{\text {nd }}$ year the crop was exposed to higher minimum temperature than the $1^{\text {st }}$ year which led to higher crop height, dry matter accumulation and nodule number in $2^{\text {nd }}$ year crops.

During the reproductive phase the $2^{\text {nd }}$ year crop was exposed to higher minimum temperature which led to flower drop, drying of the floral part and ultimate reduction of yield (Tzudir et al., 2014). Erskine et al., (1989) observed that the productivity was related to the temperature during reproductive period. Summerfield et al., (1989) observed that the higher temperature during the reproductive phase accelerated progress towards reproductive maturity and reduced seed yield. In the present experiment the minimum temperature during the reproductive phase in the $1^{\text {st }}$ year ranged in between 11.2 and $14.1{ }^{\circ} \mathrm{C}$ where as in the $2^{\text {nd }}$ year, it was in between 14.1 and 16.8 degree Celsius; there was an approximate rise of $3{ }^{\circ} \mathrm{C}$ in the $2^{\text {nd }}$ year. The maximum productivity of lentil would be achieved if the maximum and minimum temperatures ranged from 24.6 to $28.6{ }^{\circ} \mathrm{C}$ and 10.1 to $10.9{ }^{\circ} \mathrm{C}$ during $100 \%$ flowering stage.

In the Gangetic Plain of West Bengal four pre released varieties, PL 129, L 4706, SKUA-L9 and IPL 325 may be selected for their better productivity. Sowing time should be selected in such manner that the flowering stage will be reached when the maximum and minimum temperatures will hover around 24.6 to 28.6 ${ }^{\circ} \mathrm{C}$ and 10.1 to $10.9^{\circ} \mathrm{C}$ respectively. 


\section{References}

Alam, M.Z., Haider SA and Paul NK 2004. Influence of different irrigation regeson the phenology and accumulate heat units in four bread wheat cultivars. Pakistan Journal of Biological Science. 7(9): 1477-1480

Bhattacharya, A., 2009. Yield determination in lentil under non-irrigated and irrigated conditions: phenology, dry matter distribution and yield attributes. Indian Journal of Agricultural Research. 43(1): 1-9

Erksine, W., Adham, Y. and Holly, L. 1989. Geographic distribution of variation in quantitative traits in a world lentil collection. Euphytica. 43: 97-103.

He, Z.H., and Rajaram, S. 1993. Differential responses of bread wheat character to high temperature. Euphytica. 72(3): 197-203.
Hundal, S. S., and Kaur, P. 2007. Climate variability and its impact on cereal productivity in Indian Punjab. Current Science. 29(3): 631-635.

Nath, R., Chakraborty, P.K. and Chakraborty, A. 1999. Requirement of growing degree days, photothermal unit and heliothermal unit for different phenophases of sesame (Sesamum indicum L.) at different swoing dates. Indian Agriculturist. 43(3-4): 127-134

Parya, M., Nath, R., Mazumder, D. and Chakraborty P.K. 2010. Effect of thermal stress on wheat productivity in West Bengal. Journal of Agro meteorology. 12 (2): 217-220.

Summerfield, R.J., Roberts E. H., Erskine, W. and Ellis, R. H. 1985. Effects of temperature and photoperiod on flowering in lentils (Lens culinaris medic.). Annals of Botany, 56: 659-671.

\section{How to cite this article:}

Milan Kanti Kundu, Sanjib Kumar Mandi, M.D. Hasim Reja, Srijani Maji and Rajib Nath. 2017. Evaluation of Pre-Released Bold Seeded Lentil Varieties on Different Weather Factors for Growth and Yield Potentiality in the New Alluvial Zone of West Bengal. Int.J.Curr.Microbiol.App.Sci. 6(9): 758-771. doi: https://doi.org/10.20546/ijcmas.2017.609.094 